\title{
Contingency-Constrained Optimal Power Flow Using Simplex-Based Chaotic-PSO Algorithm
}

\author{
Zwe-Lee Gaing and Chia-Hung Lin \\ Department of Electrical Engineering, Kao-Yuan University, Kaohsiung City 821, Taiwan \\ Correspondence should be addressed to Zwe-Lee Gaing, zlgaing@cc.kyu.edu.tw
}

Received 26 September 2010; Revised 18 February 2011; Accepted 25 April 2011

Academic Editor: Chuan-Kang Ting

Copyright ( 2011 Z.-L. Gaing and C.-H. Lin. This is an open access article distributed under the Creative Commons Attribution License, which permits unrestricted use, distribution, and reproduction in any medium, provided the original work is properly cited.

\begin{abstract}
This paper proposes solving contingency-constrained optimal power flow (CC-OPF) by a simplex-based chaotic particle swarm optimization (SCPSO). The associated objective of CC-OPF with the considered valve-point loading effects of generators is to minimize the total generation cost, to reduce transmission loss, and to improve the bus-voltage profile under normal or postcontingent states. The proposed SCPSO method, which involves the chaotic map and the downhill simplex search, can avoid the premature convergence of PSO and escape local minima. The effectiveness of the proposed method is demonstrated in two power systems with contingency constraints and compared with other stochastic techniques in terms of solution quality and convergence rate. The experimental results show that the SCPSO-based CC-OPF method has suitable mutation schemes, thus showing robustness and effectiveness in solving contingency-constrained OPF problems.
\end{abstract}

\section{Introduction}

The purpose of an optimal power flow (OPF) function is to schedule the power system controls so as to optimize the objective function while satisfying a set of nonlinear equality and inequality constraints. The equality constraints are the nodal power balance equations, while the inequality constraints are the limits of all control or dependent variables [1, 2 ]. The objective function is mainly to optimize both activepower and reactive-power dispatches. Currently, the security and optimality of system operation have been simultaneously treated for a power system economy-security control, thus adding more complexity to the system operation $[3,4]$.

In practical power system operation, the control variables in the contingency-constrained OPF (CC-OPF) problem can be divided into continuous variables, such as power output of PV-bus generator $\left(P_{G}\right)$ and PV-bus voltage $\left(V_{G}\right)$, and discrete variables, such as transformer-tap setting $\left(T_{p}\right)$ and shunt admittance of the switchable shunt capacitor/reactor $\left(Y_{h}\right)$. Therefore, the OPF problem is a highly constrained, large-dimensional, and nonconvex optimization problem with valve-point loading effects (VPLEs) of the thermal generator being taken into consideration [5-7]. The VPLEs result in the ripples in the fuel cost function, thus the number of local optima is also increased. The CC-OPF problem is represented as a nonsmooth optimization problem with equality and inequality constraints that cannot be solved by the traditional mathematical methods.

According to the economy-security tendency, performing the OPF operation, the preprotection strategies of the system and the security constraints should be taken into account. The security constraints include the transmission capacity limit and the bus-voltage limit. It is expected to establish an economy-security operation model to defense the system that may suffer contingency impacts [3-5]. In [3], the CCOPF scheduling can be undertaken to bring the system to a more acceptable level of security represented by level 1 or 2 . Regardless of whether the system is in a normal operation or contingent state, the security constraints ensure that the system can secure the operation. Thus, the aspect of system economy-security control can be carried out. However, to construct a security-constrained optimal control for a power system generation-transmission network is an extremely difficult task. Moreover, this difficulty tends to increase with growth in system size, interconnection, and other operating problems. 
Previous efforts in solving OPF problems have employed various optimization techniques, such as genetic algorithms (GA) [7-11], tabu search (TS) [12, 13], evolutionary programming (EP) $[14,15]$, differential evolution [14$16]$, and particle swarm optimization (PSO) [5, 17-21]. In particular, because of its simple concept, easy implementation, and quick convergence, PSO has by now gained much attention and has been widely employed in solving OPF problems [22-27]. However, the objective function that does not consider contingency constraints may result in improper implementation of system economy-security control. Moreover, premature convergence may result in the local optima solution obtained by PSO [27]. Studies by Higashi and Iba [26] showed that although the standard PSO discovered solutions of reasonable quality much faster than other evolutionary algorithms, it did not possess the ability of the solutions as the number of generations was increased. Consequently, the particles become stagnated after a certain number of iterations, which reveals that some particles become inactive and the search performance cannot be further improved.

Chaos is a kind of characteristic of nonlinear systems. A chaotic motion can traverse every state in a certain region by its own regularity, and every state is visited only once. Due to the unique ergodicity and special ability to avoid being trapped in local optima, chaos search is much higher in some other stochastic algorithms, even though the chaos search often needs a large number of iterations to reach the global optimum and is not effective in large searching space. Recently, several attempts for PSO using chaos methods based on logistic map were made to overcome the drawbacks of PSO technique with premature convergence [27-31].

In this paper, a chaotic PSO technique with a simplex operator (SCPSO) for solving the CC-OPF problems is proposed. The proposed SCPSO method, which involves the chaotic map and the downhill simplex search, can avoid premature convergence of PSO and escape local minima. The objective of CC-OPF with the valve-point loading effects of generators taken into consideration is not only to minimize total generation cost, but also to reduce transmission loss and improve the bus-voltage profile under normal or postcontingent state. The effectiveness of the proposed method is demonstrated in two power systems with contingency constraints, the 26-bus and the IEEE 57-bus systems, and compared with other stochastic techniques in terms of solution quality and convergence rate.

The remainder of this paper is organized as follows. Section 2 provides the formulation of CC-OPF problem. Section 3 describes the fundamentals of SCPSO approach. Section 4 explains the development of the proposed method. Numerical examples and comparisons are provided in Section 5. Finally, Section 6 outlines the conclusion and future research.

\section{Contingency-Constrained OPF Problem}

In general, the CC-OPF is a static, nonlinear, and nonconvex optimization problem, which determines a set of optimal variables from the network state, load data, and system parameters. Optimal values are computed in order to achieve a certain goal such as minimum generation cost or transmission line power loss subject to number of equality and inequality constraints.

2.1. Contingency Constraints. Contingency constraints constitute a fundamental element of economy-security control. The contingency-constrained OPF formulation can be stated as

$$
\begin{array}{ll}
\operatorname{Min}_{x, u} & f\left(x^{(0)}, u^{(0)}\right) \\
\text { s.t. } & g^{(k)}\left(x^{(k)}, u^{(k)}\right)=0, \quad \text { for } k=0,1, \ldots, N_{c} \\
& h^{(k)}\left(x^{(k)}, u^{(k)}\right) \geq 0, \quad \text { for } k=0,1, \ldots, N_{c}
\end{array}
$$

where $x$ is the set of controllable quantities in the system and $u$ is the set of dependent variables. Objective function (1) is scalar. Equalities (2) are the conventional power equations. Inequalities (3) are the limits on the control variables $x$ and the operating limits on the power system. The superscript " $o$ " represents the precontingency (basecase) state being optimized, and superscript " $k$ " $(k>0)$ represents the postcontingency states for the $N c$ contingency cases. Moreover, the equality constraints $g^{(o)}$ change to $g^{(k)}$ to reflect the outage equipment and the control variables $x^{(o)}$ responded by changing to $x^{(k)}$.

2.2. Valve-Point Loading Effect of Generator. Typically, the valve-point effects, due to wire drawing as each steam admission valve starting to open, produce ripple-like heat rate curve as in Figure 1 [7]. To model this effect, a recurring rectified sinusoid contribution is added to the second-order polynomial function to represent the input-output equation. Thus, the fuel cost functions taking into account the valvepoint effects were expressed as

$$
C_{i}\left(P_{G i}\right)=a_{i}+b_{i} P_{G i}+c_{i} P_{G i}^{2}+\left|d_{i} \cdot \sin \left(e_{i}\left(P_{G i}^{\min }-P_{G i}\right)\right)\right|,
$$

where $a_{i}, b_{i}, c_{i}, d_{i}$, and $e_{i}$ are the cost coefficients of unit $i$.

2.3. Control and Dependent Variables. In this paper, the vector of control variables is defined as $x=\left[P_{G}, V_{G}, T_{p}, Y_{h}\right]$ and the vector of dependent variables is defined as $u=$ $\left[Q_{G}, V, S\right]$, where $Q_{G}$ is the reactive power of PV-bus generator, $V$ is the PQ-bus voltage, and $S$ is the line flow in transmission line.

2.4. Objective Function. In this paper, two subproblems of CC-OPF, namely, active power dispatch and reactive power dispatch, are considered simultaneously. The former is to achieve the goal of minimum generation cost, and the latter is to achieve the goal of minimum transmission line loss and minimum bus voltage deviation. However, an advanced goal of CC-OPF should be defined not only to minimize the total generation cost but also to reduce the transmission line loss and to improve the bus-voltage profile under 


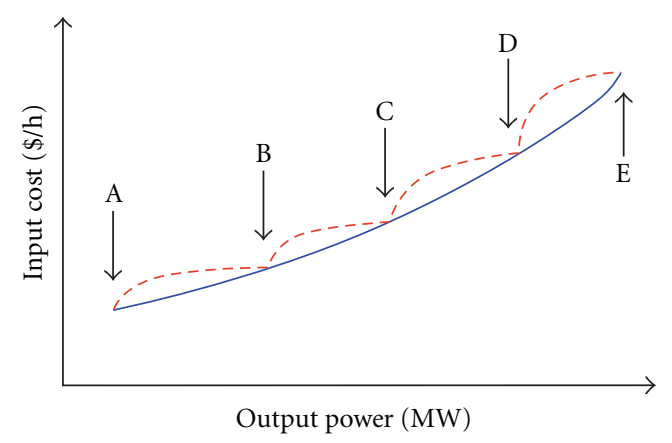

FIGURE 1: Example input-output curve with five valve points. A-E: Operating points of admission valves.

pre-contingency or post-contingency state. Minimizing the generation cost is the main objective, and reducing the transmission line loss and improving the bus voltage are also considered as objectives of CC-OPF with the valve-point loading effects of generators.

Considering the difference in homogeneity of abovementioned three objectives, however, the three objectives are the relationship of positive correlation according to the characteristic of the CC-OPF problem, so that an optimal solution obtained by the optimization algorithm can minimize the total fuel cost while involving less transmission line loss and bus voltage deviation. Hence, to convert the multiobjective problem into a single optimization problem is feasible.

Therefore, the objective function of the CC-OPF is formulated as (5) for determining an optimal setting of control variables while minimizing the objective function.

$$
f(x)=\sum_{i=1}^{N_{G}} C_{i}(x)+\sum_{l=1}^{N_{L}} \beta_{l} \cdot P_{l}(x)+\sum_{j=1}^{N_{B}} \beta_{j} \cdot\left(\left|V_{j}(x)-V_{\mathrm{ref}}\right|\right),
$$

where $N_{G}$ is the number of generator buses, $N_{B}$ is the number of buses, $N_{L}$ is the number of transmission line, and $P_{l}$ is the loss of transmission line $l$. Parameter $\beta_{l}$ is a weight factor for transferring the transmission line loss into a penalty cost, while $\beta_{j}$ is also a weight factor for transferring the voltage deviation of bus into a penalty cost. Two weight factors can be actively assigned according to the operation status, $\beta_{l}$ and $\beta_{j}$ are set to be 1.0 for transmission lines and buses energized, and 0.0 for de-energized. $V_{\text {ref }}$ is a magnitude of reference voltage; in general, $V_{\text {ref }}=1.0 \mathrm{pu}$.

(i) Equality Constraints. System power flow equations:

$$
\begin{aligned}
& P_{i}^{(k)}-\sum_{j=1}^{N_{B}}\left|Y_{i j}^{(k)}\right|\left|V_{i}^{(k)}\right|\left|V_{j}^{(k)}\right| \cos \left(\delta_{i}^{(k)}-\delta_{j}^{(k)}-\theta_{i j}^{(k)}\right)=0, \\
& Q_{i}^{(k)}-\sum_{j=1}^{N_{B}}\left|Y_{i j}^{(k)}\right|\left|V_{i}^{(k)}\right|\left|V_{j}^{(k)}\right| \sin \left(\delta_{i}^{(k)}-\delta_{j}^{(k)}-\theta_{i j}^{(k)}\right)=0 .
\end{aligned}
$$

(ii) Inequality Constraints.

(1) Active and reactive power limits of generators:

$$
\begin{gathered}
P_{G i}^{\min } \leq P_{G i}^{(k)} \leq P_{G i}^{\max }, \quad i \in N_{G}, \\
Q_{G i}^{\min } \leq Q_{G i}^{(k)} \leq Q_{G i}^{\max }, \quad i \in N_{G} .
\end{gathered}
$$

(2) Bus-voltage limit:

$$
V_{j}^{\min } \leq V_{j}^{(k)} \leq V_{j}^{\max }, \quad j \in N_{B} .
$$

(3) Transmission capacity limit:

$$
\left|S_{m}^{(k)}\right| \leq S_{m}^{\max }, \quad m \in N_{E}
$$

(4) Transformer-tap setting limit:

$$
T_{p n}^{\min } \leq T_{p n}^{(k)} \leq T_{p n}^{\max }, \quad n \in N_{T p}
$$

(5) Operation limits of switchable capacitor/reactor devices:

$$
Y_{h k}^{\min } \leq Y_{h k}^{(k)} \leq Y_{h k}^{\max }, \quad k \in N_{S h}
$$

where $N_{E}$ is the number of network branches, $N_{T p}$ is the number of transformer branches, and $N_{S h}$ is the number of the reactive power source installation buses.

Therefore, the contingency-constrained OPF problem must be solved subject to both pre-contingency and postcontingency constraints of the selected contingency cases.

\section{Chaotic Particle Swarm Optimization with Simplex Operator}

\subsection{Chaotic Particle Swarm Optimization}

(i) Classical PSO. PSO as an optimization tool provides a population-based search procedure in which individuals (called particles) change their positions (coordinates) over time. In a PSO system, particles fly around in a $D$-dimensional search space. During flight, each particle adjusts its position according to its own experience and the experience of neighboring particles, making use of the best position encountered by itself and its neighbors.

The particle swarm works by adjusting trajectories through manipulation of each coordinate of a particle. Let $x_{i}=\left(x_{i 1}, x_{i 2}, \ldots, x_{i D}\right)$, and $v_{i}=\left(v_{i 1}, v_{i 2}, \ldots, v_{i D}\right)$ denote the positions and the corresponding flight speed (velocity) of the particle $i$ in a continuous search space, respectively. 
The particles are manipulated according to the following equations [11].

$$
\begin{aligned}
v_{i}^{(t+1)}= & w^{(t)} v_{i}^{(t)}+c_{1} \cdot r_{1} \cdot\left(x_{\text {gbest }}^{(t)}-x_{i}^{(t)}\right) \\
& +c_{2} r_{2}\left(x_{\text {pbest }, i}^{(t)}-x_{i}^{(t)}\right), \\
x_{i}^{(t+1)}= & x_{i}^{(t)}+v_{i}^{(t+1)},
\end{aligned}
$$

where $t$ : pointer of iterations (generations), $w$ : inertia weight factor, $c_{1}, c_{2}$ : acceleration constant, $r_{1}, r_{2}$ : uniform random value in the range $[0,1], v_{i}^{(t)}$ : velocity of particle $x_{i}$ at iteration $t$, and $\left|v_{i}^{(t)}\right| \leq v_{i}^{\max }$, where $v_{i}^{\max }$ is the maximum velocity limits of $x_{i}, x_{i}^{(t)}$ : current position of particle $i$ at iteration $t, x_{p \text { best }, i}^{(t)}$ : the previous best position of particle $x_{i}$ at iteration $t, x_{g \text { best }}^{(t)}$ : the best position among all individuals in the population at iteration $t, v_{i}^{(t+1)}$ : new velocity of particle $x_{i}$, and $x_{i}^{(t+1)}$ : new position of particle $x_{i}$.

In (13), the proper selection of inertia weight $w$ will provide a balance between global explorations and local exploitation, thus requiring fewer iterations on average to find an optimal solution. In general, a decreasing linearly inertia weight $w$ is set (15).

$$
w^{(t)}=w^{\max }-\frac{w^{\max }-w^{\min }}{t_{\max }} \times t,
$$

where $t_{\max }$ is the maximum number of iterations (generations) and $t$ is the current number of iterations.

(ii) Chaotic-PSO. The advantages of the classical PSO are simple concept, easy implementation, robustness to control parameters, and computational efficiency. However, it depends greatly on its parameters and exists as the premature convergence phenomenon, especially in solving complex multihump problems with equality and inequality constraints. Conversely, owing to the properties of unique ergodicity, inherent stochastic property, and irregularity of chaos, a chaotic search can traverse every state in a certain space by its own regularity and visit every state once only, which helps avoid being trapped in local optima. Thus, a chaotic search has a much higher precision than some other stochastic algorithms [27-30].

(iii) Chaotic Map. To enrich the search behavior and avoid the premature phenomenon of PSO in solving OPF problems, incorporating a chaotic search into PSO to construct a chaotic PSO is proposed. The chaotic search algorithm is developed from the chaotic evolution of variables. Two wellknown chaotic maps, logistic map and tent map, are the most common maps used in chaotic searches [27-29, 31].

The logistic map is defined by

$$
z^{n+1}=4 z^{n}\left(1-z^{n}\right), \quad 0 \leq z^{0} \leq 1, n=0,1,2, \ldots
$$

The feature of the logistic map is that a small difference in the initial value of the chaotic variable would result in a considerable difference in its long-time behaviors; a chaotic
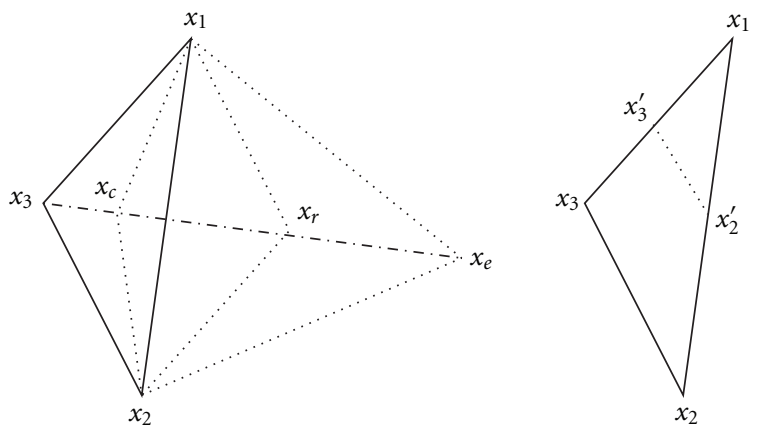

Figure 2: Four operations in downhill simplex method. $\left(x_{r}\right.$ : reflection, $x_{e}$ : expansion, $x_{c}$ : contraction, $x_{2}^{\prime}, x_{3}^{\prime}$ : shrinkage).

variable can travel ergodically over the entire search space $[18,19]$.

The tent map is defined by

$$
z^{n+1}=\mu\left(1-2\left|z^{n}-0.5\right|\right), \quad 0 \leq z^{0} \leq 1, \mu \in[0,1] .
$$

Similar to the uniform distribution function in the interval $[0,1]$, the tent map has outstanding advantages and faster iterative speed than the logistic map, and therefore, it has excellent characteristic of ergodicity. In this paper, the tent map is employed to generate chaotic variables for enriching the search behavior.

\subsection{Simplex Operator}

(i) Downhill Simplex Method. A local search method called the Downhill simplex method is one of the most popular derivate-free nonlinear optimization algorithms [32, 33]. In the $n$-dimensional space, a simplex is a polyhedron with $n+1$ vertices. The method iteratively updates the worst point by four operations process: reflection, expansion, contraction, and shrinkage that are shown in Figure 2. Reflection involves moving the worst point (vertex) of simplex to a point reflected through the remaining $n$ points. If this point is better than the best point, then the method attempts to expand the simplex along this line. This operation is called expansion. On the other hand, if the new point is not much better than the previous point, then the simplex is contracted along one dimension from the worst point. The procedure is called contraction. Moreover, if the new point is worse than the previous points, the simplex is contracted along all dimensions toward the best point and steps down the valley. The procedure is called shrinkage.

In each iteration, new points are computed, along with their function values, to form a new simplex. By repeating this series of operations, the method finds the optimal solution.

(ii) Simplex Search Algorithm. The calculation procedures of the simplex search algorithm (SSA) are described as follows [32-35]. The flowchart of SSA is shown in Figure 3.

(1) Order and relabel the $n+1$ points as $x_{1}, x_{2}, \ldots, x_{n+1}$ so that $f\left(x_{1}\right) \leq f\left(x_{2}\right) \leq \cdots \leq f\left(x_{n+1}\right)$. 


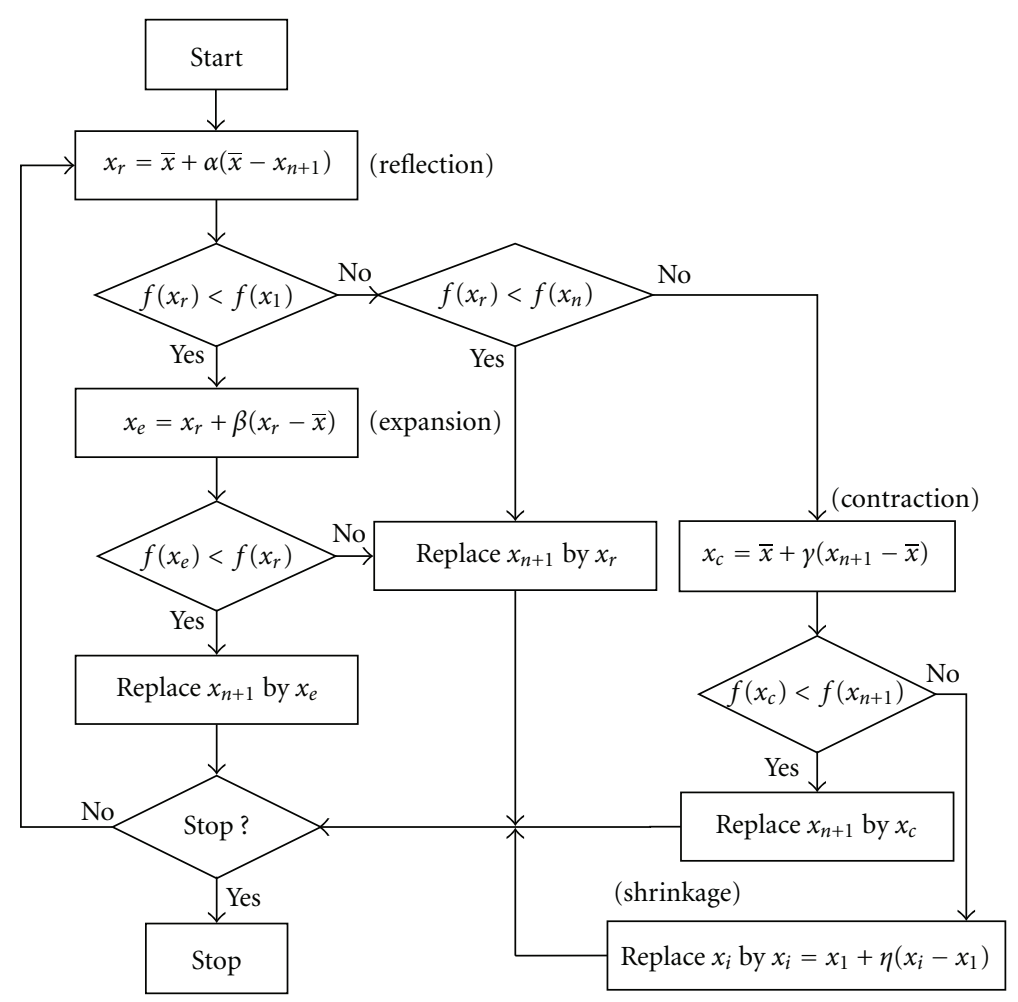

FIGURE 3: Flowchart of SSA.

(2) Generate a trial point $x_{r}$ by reflection, such that

$$
x_{r}=\bar{x}+\alpha\left(\bar{x}-x_{n+1}\right),
$$

where $\bar{x}$ is the centroid of the $\mathrm{n}$ best points in the vertices of the simplex. If $f\left(x_{1}\right) \leq f\left(x_{r}\right) \leq f\left(x_{n}\right)$, replace $x_{n+1}$ by $x_{r}$.

(3) If $f\left(x_{r}\right)<f\left(x_{1}\right)$, generate a new point $x_{e}$ by expansion, such that

$$
x_{e}=\bar{x}+\beta\left(x_{r}-\bar{x}\right) .
$$

If $f\left(x_{e}\right)<f\left(x_{r}\right)$, replace $x_{n+1}$ by $x_{e}$, otherwise replace $x_{n+1}$ by $x_{r}$.

(4) If $f\left(x_{r}\right) \geq f\left(x_{n}\right)$, generate a new point $x_{c}$ by contraction, such that

$$
x_{c}=\bar{x}+\gamma\left(x_{n+1}-\bar{x}\right) .
$$

If $f\left(x_{c}\right)<f\left(x_{n+1}\right)$, replace $x_{n+1}$ by $x_{c}$.

(5) If $f\left(x_{c}\right) \geq f\left(x_{n+1}\right)$, shrink along all dimensions toward $x_{1}$, such that

$$
x_{i}^{\prime}=x_{1}+\eta\left(x_{i}-x_{1}\right) .
$$

Replace $x_{i}$ by $x_{i}^{\prime}$. Evaluate $f$ at the $n$ new vertices.

(6) Order and relabel the vertices of the new simplex as $x_{1}, x_{2}, \ldots, x_{n+1}$, such that $f\left(x_{1}\right) \leq f\left(x_{2}\right) \leq \cdots \leq$ $f\left(x_{n+1}\right)$. If the stopping criterion is satisfied, then stop. Otherwise go to step 2.
In general, four scalar parameters, coefficients of reflection $\alpha$, expansion $\beta$, contraction $\gamma$, and shrinkage must be specified to define a complete downhill simplex method $\eta$. Many articles have reported that coefficient values of $\alpha=1.0$, $\beta=2.0, \gamma=0.5$, and $\eta=0.5$ are used [34]. Figure 2 shows the reflection, expansion, contraction, and shrinkage points for a simplex in two dimensions using the values of abovementioned coefficients.

3.3. Chaotic-PSO with Simplex Operator. To enhance the exploration-exploitation ability of the chaotic PSO method, the chaotic-PSO with simplex operator is included. The proposed method is made up of two parts. One is the chaotic-PSO that engages in global exploration, the other is the simplex search for increasing the local exploitation that can escape the local minimum and accelerate the converge process. The calculation procedures of the proposed SCPSO algorithm are described as follows.

(1) Set the $t_{\max }$ and generate the initial population. Compare the fitness of each particle to obtain its $x_{p \text { best }}$. The best $x_{p \text { best }}$ is denoted $x_{\text {gbest }}$.

(2) Use the tent map $(\mu=1)$ to generate the chaotic variables according to (22).

$$
\begin{aligned}
z_{i}^{(k)} & =\frac{x_{i}^{(t)}-x_{i}^{\min }}{x_{i}^{\max }-x_{i}^{\min }}, \\
z_{i}^{(k+1)} z_{i}^{(k+1)} & =\left(1-2\left|z_{i}^{(k)}-0.5\right|\right), \quad i=0,1,2, \ldots, D .
\end{aligned}
$$




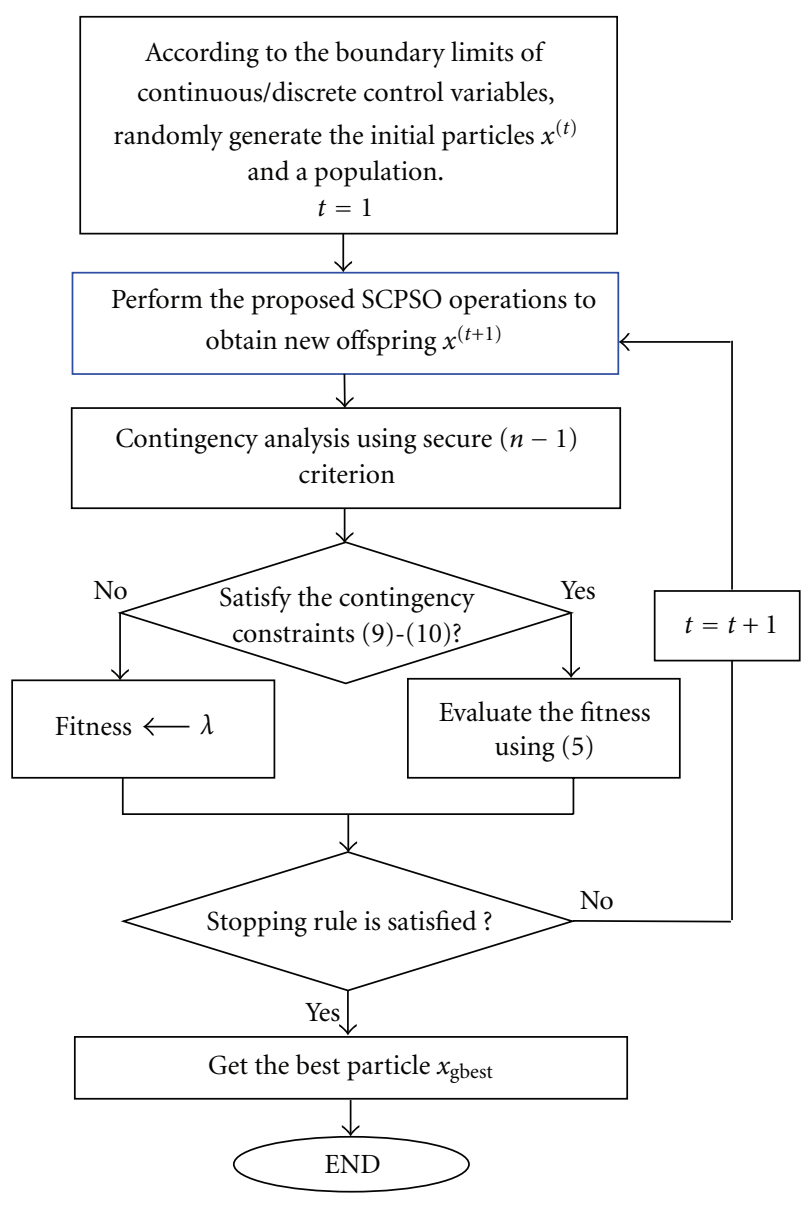

FIGURE 4: Operating procedures of the proposed SCPSO-based CC-OPF method.

Map the chaotic variables $z_{i}^{(k+1)}$ into the search range of decision variables $x_{i}^{(k+1)}$.

$x_{i}^{(t)}=x_{i}^{\min }+z_{i}^{(k+1)}\left(x_{i}^{\max }-x_{i}^{\min }\right), \quad i=0,1,2, \ldots, D$.

(3) Update the particle's velocity $v^{(t+1)}$ and position $x^{(t+1)}$ according to (13) and (14), respectively. In addition, $\left|v^{(t+1)}\right| \leq v^{\max }$.

Evaluate the fitness $f^{(t+1)}$ for each update particle.

Update $x_{p \text { best }}^{(t+1)}$ and $x_{g \text { best }}^{(t+1)}$ if needed.

(4) Order and relabel all new particles (new offsprint) $x^{(t+1)}$ according to their fitness. Apply a small number of iterations of simplex search to improve all new particles in the population.

(5) Let $t:=t+1$ and repeat Steps 2-5 until the stopping criterion $\left(t>t_{\max }\right)$ is met.

(6) The latest $x_{\text {gbest }}$ is the optimal solution.

\section{Development of the Proposed Method}

4.1. Representation of Particle. In this paper, the particle comprises both continuous control variables $x_{c}$ and discrete control variables $x_{d}$. A particle $x$ is a mixed-integer structure, that is, $x=\left[x_{c}, x_{d}\right]=\left[P_{G}, V_{G}, T_{p}, Y_{h}\right]$. The physical variables are encoded as follows.

(1) Continuous variable $x_{c i}$ taking the real value in the interval $\left[x_{c i}^{\min }, x_{c i}^{\max }\right], x_{c i} \in\left[P_{G}, V_{G}\right]$.

(2) Discrete variable $x_{d i}$ taking the decimal integer value $n_{i}$ in the interval $\left[0, \ldots, M_{i}\right], x_{d i} \in\left[T_{p}, Y_{h}\right]$.

$$
M_{i}=\operatorname{INT}\left(\frac{x_{d i}^{\max }-x_{d i}^{\min }}{\mathrm{ST}_{i}}\right),
$$

where $\mathrm{ST}_{i}$ is the adjustable step size of the discrete control variable $x_{d i}$. INT $(\cdot)$ is the operator rounding the variable to the nearest integer. To transform a discrete variable $x_{d i}$ into a practical control value is as in (25).

$$
x_{d i}=x_{d i}^{\min }+n_{i} \cdot \mathrm{ST}_{i}
$$

4.2. SCPSO-Based CC-OPF. As mentioned above, the objective of CC-OPF is not only to minimize total operation cost, but also to enhance transmission security, reduce transmission loss, and improve the bus-voltage profile under pre-contingency or post-contingency state. The search procedures of the SCPSO-based CC-OPF method are shown 


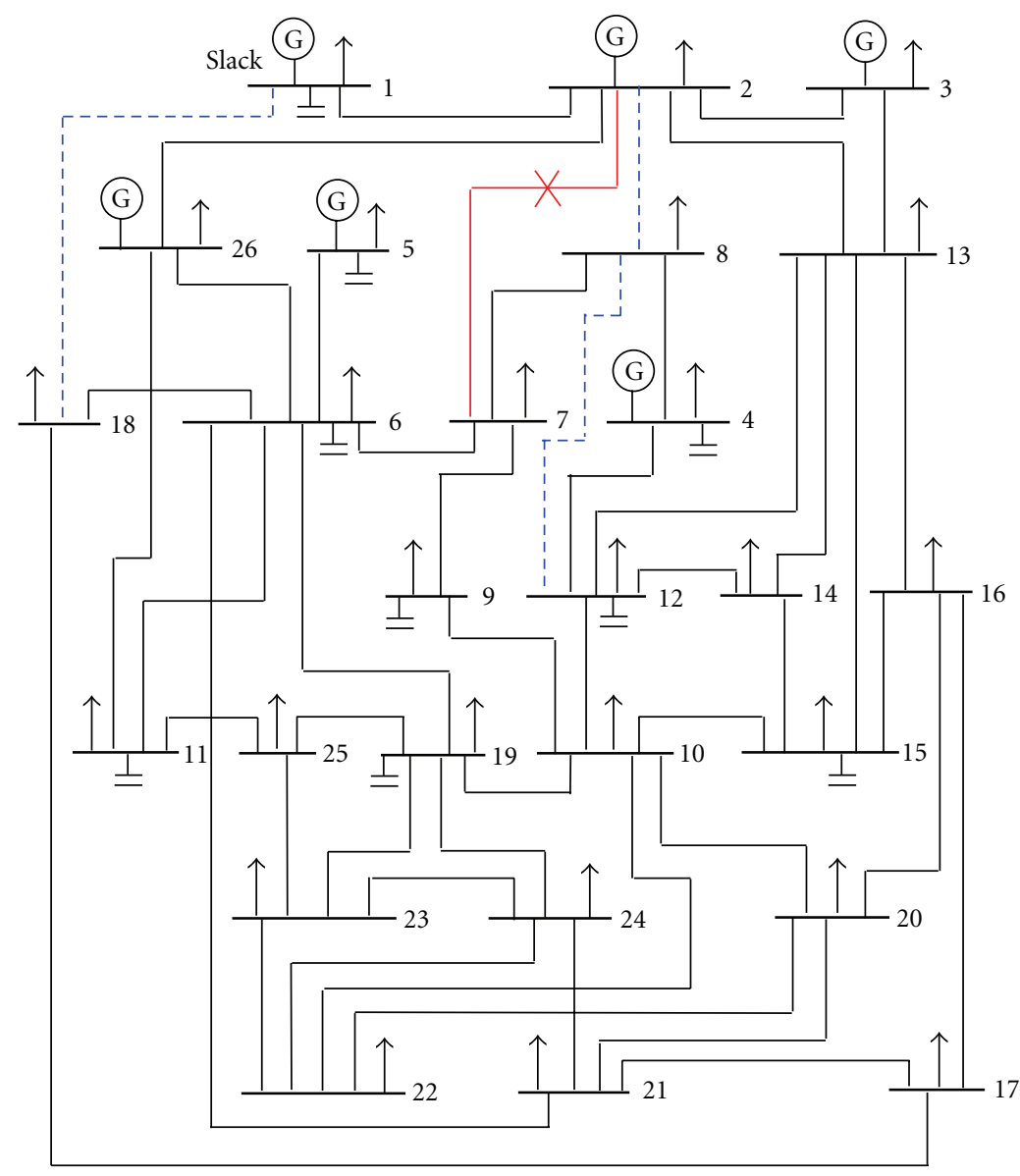

FIgURE 5: One-lone diagram of 26-bus system.

TABLE 1: Generating unit capacity and coefficients in 26-bus System.

\begin{tabular}{lcccccccccc}
\hline Bus no. & $P_{i}^{\min }$ & $P_{i}^{\max }$ & $Q_{i}^{\min }$ & $Q_{i}^{\max }$ & $S_{i}^{\max }$ & $a_{i}$ & $b_{i}$ & $c_{i}$ & $d_{i}$ & $e_{i}$ \\
\hline 1 & 100 & 500 & 80 & 300 & 550 & 240 & 7.0 & 0.0070 & 100 & 0.0545 \\
2 & 50 & 200 & 40 & 250 & 300 & 200 & 10.0 & 0.0095 & 80 & 0.0825 \\
3 & 80 & 300 & 40 & 150 & 350 & 220 & 8.5 & 0.0090 & 80 & 0.0710 \\
4 & 50 & 150 & 40 & 80 & 200 & 200 & 11.0 & 0.0090 & 50 & 0.0930 \\
5 & 50 & 200 & 40 & 160 & 250 & 220 & 10.5 & 0.0080 & 80 & 0.0825 \\
26 & 50 & 120 & 15 & 50 & 150 & 190 & 12.0 & 0.0075 & 50 & 0.0900 \\
\hline
\end{tabular}

in Figure 4. The objective function in (5) is employed as a fitness function. If a particle $x$ is a feasible solution and satisfies all constraints, its fitness will be measured by (5). Otherwise, its fitness will be penalized with a very large positive constant $\lambda$ (i.e., the dependent variable violates either the equality constraints (6) or the inequality constraints (8)-(10)).

\section{Numerical Examples and Results}

When the constraints of the valve-point loading effects of generators are considered, the OPF problem becomes nonconvex and may thus degrade the quality of solution and convergence rate. To verify the feasibility and robustness of the proposed SCPSO-based OPF method, a 26-bus and an
IEEE 57-bus systems were tested. The proposed method was compared with other stochastic methods, such as chaoticbased PSO (CPSO) [27], PSO with Gaussian mutation (MPSO) [25], improved PSO with linearly decreasing inertia weight (IPSO) [22], hybrid genetic algorithm (HGA) [11] and differential evolution (DE) [16], in terms of solution quality and computation efficiency using the same fitness function and particle definition. The maximum number of iterations for all the algorithms is set to 100 .

\subsection{Description of Study Systems}

(i) 26-Bus System. The system that contains six thermal units, 26 buses, and 46 transmission lines is shown in 
TABLE 2: Generating unit capacity and coefficients in IEEE 57-bus System.

\begin{tabular}{lcccccccccc}
\hline Bus no. & $P_{G i}^{\min }$ & $P_{G i}^{\max }$ & $Q_{G i}^{\min }$ & $Q_{G i}^{\max }$ & $S_{G i}^{\max }$ & $a_{i}$ & $b_{i}$ & $c_{i}$ & $d_{i}$ \\
\hline 1 & 100 & 500 & -200 & 300 & 550 & 240 & 7.0 & 0.007 & 100 & 0.0545 \\
2 & 50 & 150 & -50 & 60 & 200 & 200 & 11.0 & 0.009 & 50 & 0.0930 \\
3 & 50 & 150 & -50 & 60 & 200 & 200 & 11.0 & 0.009 & 50 & 0.0930 \\
6 & 50 & 120 & -40 & 50 & 180 & 190 & 12.0 & 0.0075 & 50 & 0.0900 \\
8 & 80 & 300 & -150 & 200 & 350 & 220 & 8.5 & 0.009 & 80 & 0.0710 \\
9 & 50 & 120 & -40 & 50 & 180 & 190 & 12.0 & 0.0075 & 50 & 0.0900 \\
12 & 80 & 300 & -150 & 200 & 350 & 220 & 8.5 & 0.009 & 80 & 0.0710 \\
\hline
\end{tabular}

TABLE 3: System state under normal operation and post-contingency.

\begin{tabular}{llccr}
\hline \multirow{2}{*}{ Study system } & \multicolumn{2}{c}{ Normal operation (Pre-contingency) } & \multicolumn{2}{c}{ Postcontingency } \\
& Line & Line flow $(\mathrm{Mva})$ & Line outage & $\mathrm{L}_{1-18}, \mathrm{~L}_{2-8}, \mathrm{~L}_{8-12}$ \\
\hline 26-bus & $\mathrm{L}_{2-7}$ & 74.31 & $\mathrm{~L}_{2-7}$ & $\mathrm{~L}_{1-16}, \mathrm{~L}_{2-3}$ \\
IEEE 57-bus & $\mathrm{L}_{1-17}$ & 100.82 & $\mathrm{~L}_{1-17}$ & \\
\hline
\end{tabular}

Figure 5 [12]. The load demand is $1263 \mathrm{MW}$. The detailed characteristics of the six thermal units with the valve-point loading effects are given in Table 1. Let Bus 1 denote the slack bus; the bus data, branch data, transformer-tap data, and shunt-capacitor bank data of the system are shown in [16].

The system has a total of 27 control variables as follows: 5 unit active power outputs, 6 generator-bus voltage magnitudes, 7 transformer-tap settings, and 9 var-injection values of shunt capacitor. The adjustable range of the transformertap is from $0.9 \mathrm{pu}$ to $1.1 \mathrm{pu}$, and the shunt admittance of shunt capacitor is 0.0 to $\mathrm{j} 0.05 \mathrm{pu}$. The adjustable step size is from $0.01 \mathrm{pu}$ in the transformer-tap settings, and the changing step size is j0.005 pu in the shunt admittance. According to (24), the $M$ values of the two discrete variables above are 20 and 10, respectively. The upper and lower limits of the generator-bus and load-bus voltages are $0.95 \mathrm{pu}$ and $1.05 \mathrm{pu}$, respectively.

(ii) IEEE 57-Bus System. The IEEE 57-bus system contains seven thermal units, 57 buses and 46 transmission lines. The load demand is $1250.8 \mathrm{MW}$. The detailed characteristics of the seven thermal generators with the valve-point loading effects are given in Table 2. Bus 1 is the swing bus.

The system has a total of 31 control variables as follows: 6 active power outputs, 7 generator-bus voltage magnitudes, 15 transformer-tap settings, and 3 var-injection values of shunt capacitor. Because the adjustable range of the transformertap is $0.9-1.1 \mathrm{pu}$, and the shunt admittance ranges from 0.0 to $0.1 \mathrm{pu}$, the adjustable step size in the transformer-tap settings is $0.01 \mathrm{pu}$, and the changing step size in the shunt admittance is $0.005 \mathrm{pu}$. The $M$ values of the two discrete variables above are 20 and 10, respectively. The upper and lower limits of the generator-bus and load-bus voltages are $0.9 \mathrm{pu}$ and $1.1 \mathrm{pu}$, respectively.

5.2. Selected Contingency Event. Table 3 shows two states of the study systems. One is the normal operation (precontingency), and the other is the post-contingency with a selected contingency occurring. In 26-bus system, the power flow on transmission line $\mathrm{L}_{2-7}$ is about $74.31 \mathrm{Mva}$ in normal economic operation. From the results of contingency selecting, one of the most critical faults is proven line $\mathrm{L}_{2-7}$ outage. When line $\mathrm{L}_{2-7}$ faulted, three lines $\left(\mathrm{L}_{1-18}, \mathrm{~L}_{2-8}\right.$, and $\mathrm{L}_{8-12}$ ) were overloaded, as shown in Figure 5. In the IEEE 57-bus system, the power flow on transmission line $\mathrm{L}_{1-17}$ is about 100.82 Mva under normal operation. When $\mathrm{L}_{1-17}$ faulted, two lines ( $\mathrm{L}_{1-16}$ and $\mathrm{L}_{2-3}$ ) were overloaded.

5.3. Parameters of Algorithms. Through repeated experiments, the suitable parameters of the proposed SCPSO method in Table 4 can be used. The population size is set to be 50 and the number of iterations is set to be 100 . Those coefficients of reflection $\alpha$, expansion $\beta$, contraction $\gamma$, and shrinkage $\eta$ in SSA method are 1.0, 2.0, 0.5, and 0.5 , respectively. Maximum number of iterations for the SSA method is set to be 10 .

5.4. Experimental Results. In each study system, a total of 30 trials were performed. The simulation results are summarized in Table 5. The optimal settings of control variables obtained by the four proposed methods are shown in Table 6.

In Table 5, three performance indexes, namely the distribution region $(\Delta f)$, the mean value $(\mu)$, and the standard deviation $(\sigma)$ are employed to verify the robustness of the proposed method. Best fitness obtained by each trial was recorded. The proposed indexes were employed to evaluate the effectiveness of the proposed method in solving the CCOPF problem.

$$
\begin{gathered}
\Delta f=f_{\max }-f_{\min }, \\
\mu=\frac{1}{n} \sum_{i=1}^{n} f_{i}, \\
\sigma=\sqrt{\frac{1}{n} \sum_{i=1}^{n}\left(f_{i}-\mu\right)^{2},}
\end{gathered}
$$


TABLE 4: Parameters of proposed algorithms.

\begin{tabular}{lcc}
\hline PSO Parameters & SCPSO, CPSO, MPSO, IPSO \\
\hline$w$ & & $0.9-0.4$ \\
$c_{1}$ & & 1.05 \\
$c_{2}$ & & 1.05 \\
$v_{c i}^{\max }$ & HGA & $x_{c i}^{\max } / 2$ \\
$v_{d i}^{\max }$ & 0.6 & $M_{i} / 2$ \\
\hline HGA and DE Parameters & 0.05 & DE \\
\hline CR (crossover rate) & & 0.5 \\
$P_{m}$ (mutation rate) & - \\
$F$ (scaling factor) & 0.5 \\
\hline
\end{tabular}

TABLE 5: Comparisons of four methods in two study Systems.

\begin{tabular}{|c|c|c|c|c|c|c|}
\hline \multirow{2}{*}{ Study system } & \multirow{2}{*}{ Method } & \multicolumn{2}{|c|}{ Fitness } & \multicolumn{3}{|c|}{ Performance index } \\
\hline & & $f_{\min }$ (best) & $f_{\max }$ (worst) & $\Delta f$ & $\mu$ & $\sigma$ \\
\hline \multirow{6}{*}{ 26-bus } & SCPSO & 15499 & 15587 & 87 & 15573 & 14.3111 \\
\hline & CPSO & 15503 & 15612 & 109 & 15578 & 26.2314 \\
\hline & MPSO & 15545 & 15633 & 88 & 15618 & 27.1884 \\
\hline & IPSO & 15552 & 15658 & 106 & 15619 & 27.2129 \\
\hline & HGA & 15568 & 15857 & 189 & 15658 & 29.1129 \\
\hline & $\mathrm{DE}$ & 15575 & 15714 & 139 & 15668 & 30.4691 \\
\hline \multirow{6}{*}{ IEEE 57-bus } & SCPSO & 15426 & 15504 & 78 & 15447 & 16.1596 \\
\hline & CPSO & 15435 & 15550 & 109 & 15471 & 30.1171 \\
\hline & MPSO & 15436 & 15546 & 110 & 15479 & 40.2011 \\
\hline & IPSO & 15443 & 15561 & 118 & 15494 & 37.1354 \\
\hline & HGA & 15453 & 15573 & 120 & 15511 & 47.3282 \\
\hline & $\mathrm{DE}$ & 15470 & 15579 & 109 & 15518 & 39.5413 \\
\hline
\end{tabular}

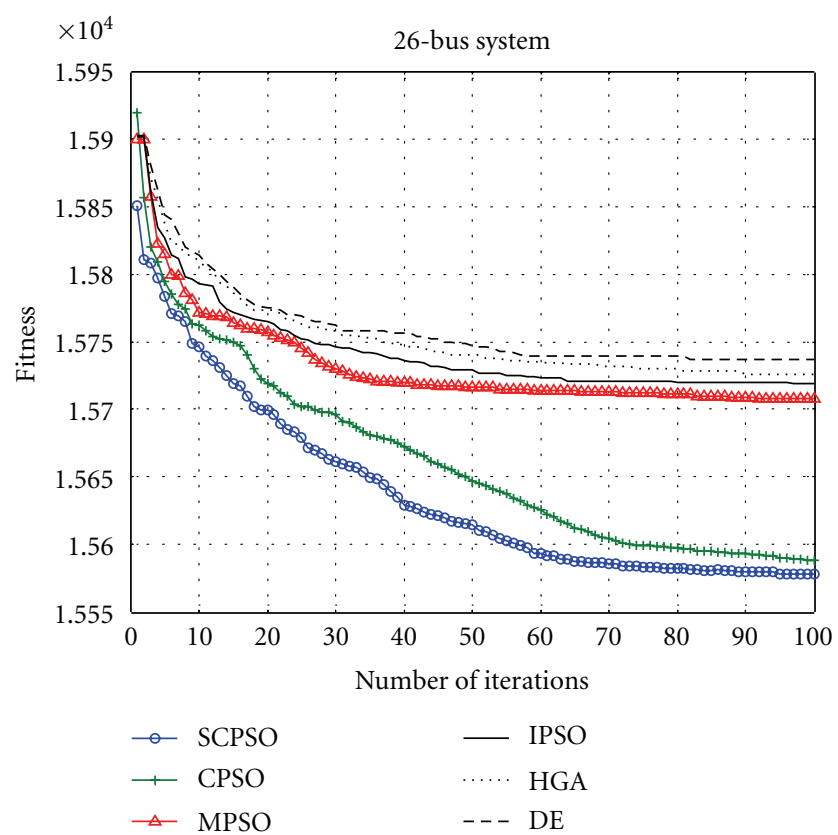

(a)

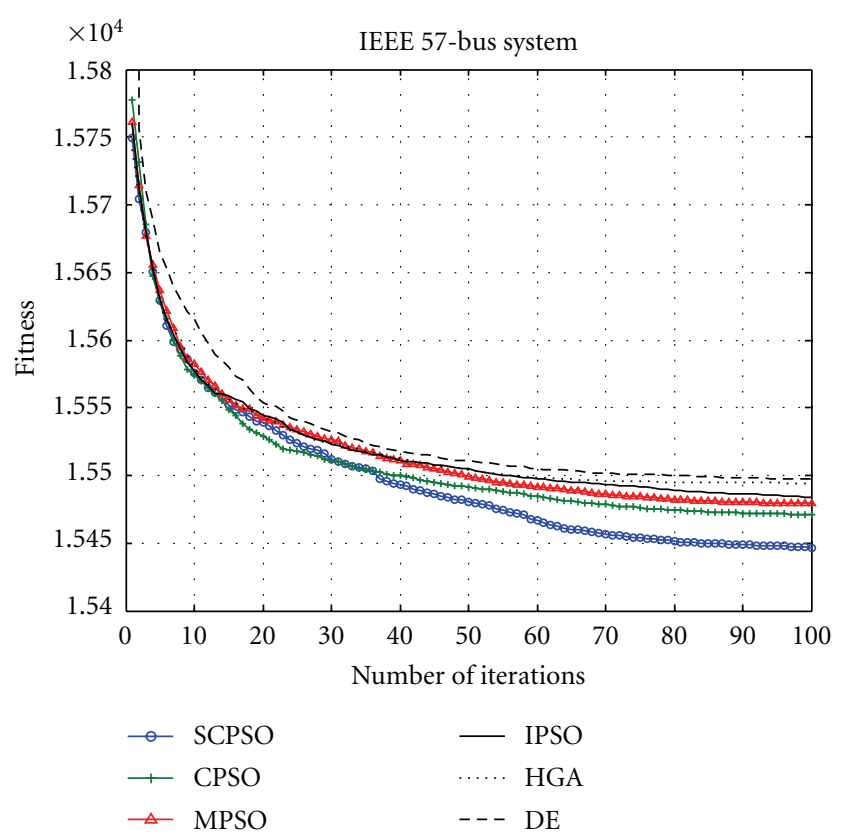

(b)

FIGURE 6: Convergence tendency. (a) Convergence tendencies of average fitness over 30 trials in 26-bus system. (b) Convergence tendencies of average fitness over 30 trials in IEEE 57-bus system. 
TABLE 6

(a) Optimal settings of control variables in 26-bus system

\begin{tabular}{|c|c|c|c|c|c|c|}
\hline Control variable & SCPSO & CPSO & MPSO & IPSO & HGA & $\mathrm{DE}$ \\
\hline$P_{G 1}$ & 446.1746 & 448.1017 & 445.7566 & 453.3504 & 451.8483 & 452.6614 \\
\hline$P_{G 2}$ & 164.7378 & 166.1304 & 200.0000 & 165.0258 & 166.6793 & 165.7782 \\
\hline$P_{G 3}$ & 258.4341 & 258.1863 & 262.1278 & 259.9621 & 257.4760 & 256.2267 \\
\hline$P_{G 4}$ & 149.5769 & 149.9920 & 118.0309 & 136.5176 & 144.1294 & 142.0623 \\
\hline$P_{G 5}$ & 164.5429 & 164.4053 & 200.0000 & 164.5045 & 166.6446 & 163.0210 \\
\hline$P_{G 26}$ & 91.1452 & 87.9374 & 50.0000 & 103.2680 & 94.6340 & 102.8937 \\
\hline$V_{1}$ & 1.0327 & 1.0279 & 1.0411 & 1.0404 & 1.0403 & 1.0286 \\
\hline$V_{2}$ & 0.9920 & 1.0500 & 1.0088 & 1.0174 & 1.0186 & 1.0190 \\
\hline$V_{3}$ & 1.0345 & 1.0201 & 1.0211 & 0.9943 & 0.9959 & 0.9907 \\
\hline$V_{4}$ & 1.0346 & 0.9823 & 1.0004 & 0.9754 & 1.0308 & 1.0320 \\
\hline$V_{5}$ & 1.0233 & 1.0101 & 0.9751 & 1.0090 & 1.0055 & 1.0053 \\
\hline$V_{26}$ & 1.0208 & 1.0291 & 1.0248 & 1.0048 & 1.0055 & 1.0082 \\
\hline$T_{p 2-3}$ & 1.0200 & 1.0200 & 1.1000 & 1.0100 & 1.0600 & 1.0600 \\
\hline$T_{p 2-13}$ & 1.0200 & 1.0000 & 0.9700 & 1.0000 & 0.9300 & 0.9200 \\
\hline$T_{p 3-13}$ & 0.9700 & 0.9700 & 0.9500 & 0.9900 & 0.9900 & 0.9700 \\
\hline$T_{p 4-8}$ & 0.9800 & 0.9800 & 1.0200 & 1.0200 & 1.0200 & 1.0300 \\
\hline$T_{p 4-12}$ & 0.9800 & 0.9900 & 1.0400 & 1.0300 & 1.0000 & 1.0100 \\
\hline$T_{p 6-19}$ & 0.9600 & 0.9500 & 1.0100 & 0.9800 & 0.9700 & 0.9300 \\
\hline$T_{p 7-9}$ & 0.9800 & 0.9800 & 0.9500 & 0.9600 & 0.9600 & 0.9600 \\
\hline$Y_{h 1}$ & 0.0500 & 0.0000 & 0.0050 & 0.0500 & 0.0400 & 0.0500 \\
\hline$Y_{h 4}$ & 0.0300 & 0.0350 & 0.0400 & 0.0300 & 0.0300 & 0.0150 \\
\hline$Y_{h 5}$ & 0.0500 & 0.0500 & 0.0500 & 0.0400 & 0.0500 & 0.0150 \\
\hline$Y_{h 6}$ & 0.0500 & 0.0500 & 0.0150 & 0.0200 & 0.0450 & 0.0500 \\
\hline$Y_{h 9}$ & 0.0150 & 0.0400 & 0.0150 & 0.0400 & 0.0300 & 0.0250 \\
\hline$Y_{h 11}$ & 0.0250 & 0.0450 & 0.0350 & 0.0250 & 0.0350 & 0.0300 \\
\hline$Y_{h 12}$ & 0.0500 & 0.0350 & 0.0250 & 0.0500 & 0.0300 & 0.0200 \\
\hline$Y_{h 15}$ & 0.0500 & 0.0350 & 0.0100 & 0.0500 & 0.0200 & 0.0450 \\
\hline$Y_{h 19}$ & 0.0450 & 0.0500 & 0.0150 & 0.0250 & 0.0200 & 0.0500 \\
\hline Fitness $f$ & 15499 & 15503 & 15545 & 15552 & 15568 & 15575 \\
\hline$C_{i}(\$)$ & 15487 & 15491 & 15532 & 15539 & 15558 & 15562 \\
\hline$P_{\text {Loss }}(M W)$ & 11.6166 & 11.7561 & 12.9153 & 12.5594 & 12.8161 & 12.6880 \\
\hline$\sum\left|V_{i}-V_{\text {ref }}\right|$ & 0.3432 & 0.3411 & 0.5225 & 0.4599 & 0.4402 & 0.3485 \\
\hline
\end{tabular}

(b) Optimal settings of control variables in IEEE 57-bus system

\begin{tabular}{|c|c|c|c|c|c|c|}
\hline Control variable & SCPSO & CPSO & MPSO & IPSO & HGA & $\mathrm{DE}$ \\
\hline$P_{G 1}(W)$ & 330.6258 & 331.4865 & 331.3076 & 331.8135 & 330.6408 & 331.6042 \\
\hline$P_{G 2}(W)$ & 50.0000 & 56.1645 & 83.7176 & 84.4205 & 83.7784 & 83.9324 \\
\hline$P_{G 3}(W)$ & 117.5876 & 117.7136 & 117.6009 & 117.8681 & 92.7311 & 117.6796 \\
\hline$P_{G 6}(W)$ & 84.8626 & 85.8677 & 84.8836 & 87.4047 & 85.0812 & 60.2967 \\
\hline$P_{G 8}(W)$ & 300.0000 & 256.9933 & 256.9824 & 257.0185 & 257.0101 & 256.9304 \\
\hline$P_{G 9}(W)$ & 84.8851 & 119.8924 & 95.4473 & 91.9031 & 120.0000 & 120.0000 \\
\hline$P_{G 12}(W)$ & 300.0000 & 299.9529 & 300.0000 & 300.0000 & 300.0000 & 300.0000 \\
\hline$V_{G 1}$ & 1.0500 & 1.0500 & 1.0500 & 1.0500 & 1.0500 & 1.0500 \\
\hline$V_{G 2}$ & 1.0480 & 1.0495 & 1.0500 & 1.0497 & 1.0497 & 1.0500 \\
\hline$V_{G 3}$ & 1.0500 & 1.0500 & 1.0500 & 1.0498 & 1.0079 & 1.0478 \\
\hline$V_{G 6}$ & 1.0496 & 1.0462 & 1.0500 & 1.0500 & 1.0500 & 1.0500 \\
\hline$V_{G 8}$ & 1.0500 & 1.0498 & 1.0500 & 1.0500 & 1.0500 & 1.0456 \\
\hline$V_{G 9}$ & 1.0341 & 1.0446 & 1.0438 & 1.0433 & 1.0500 & 1.0500 \\
\hline
\end{tabular}


(b) Continued.

\begin{tabular}{|c|c|c|c|c|c|c|}
\hline Control variable & SCPSO & CPSO & MPSO & IPSO & HGA & $\mathrm{DE}$ \\
\hline$V_{G 12}$ & 1.0252 & 1.0500 & 1.0500 & 1.0500 & 1.0500 & 1.0498 \\
\hline$T_{p 4-18}$ & 0.9700 & 0.9600 & 0.9600 & 0.9400 & 1.0100 & 0.9600 \\
\hline$T_{p 7-29}$ & 0.9700 & 1.0300 & 1.0000 & 1.0500 & 0.9400 & 0.9400 \\
\hline$T_{p 9-55}$ & 0.9700 & 1.0300 & 0.9900 & 1.0500 & 0.9400 & 0.9400 \\
\hline$T_{p 10-51}$ & 0.9400 & 0.9400 & 0.9400 & 0.9600 & 0.9500 & 1.0100 \\
\hline$T_{p 11-41}$ & 0.9900 & 1.0300 & 0.9700 & 0.9300 & 0.9600 & 1.1000 \\
\hline$T_{p 11-43}$ & 0.9300 & 0.9200 & 0.9300 & 1.1000 & 0.9400 & 0.9200 \\
\hline$T_{p 13-49}$ & 0.9000 & 0.9000 & 0.9000 & 0.9200 & 0.9000 & 1.0100 \\
\hline$T_{p 14-46}$ & 0.9200 & 0.9200 & 0.9200 & 0.9400 & 0.9300 & 0.9800 \\
\hline$T_{p 15-45}$ & 0.9300 & 0.9400 & 0.9400 & 0.9400 & 0.9300 & 0.9600 \\
\hline$T_{p 20-21}$ & 1.0200 & 1.0400 & 1.0100 & 1.0000 & 1.1000 & 0.9400 \\
\hline$T_{p 24-25}$ & 1.0300 & 0.9500 & 1.0000 & 0.9200 & 0.9600 & 0.9300 \\
\hline$T_{p 24-26}$ & 1.0400 & 1.0900 & 1.0800 & 1.1000 & 1.0000 & 0.9500 \\
\hline$T_{p 32-34}$ & 0.9800 & 0.9600 & 0.9500 & 0.9400 & 0.9600 & 0.9000 \\
\hline$T_{p 40-56}$ & 1.0300 & 1.0100 & 1.0400 & 1.0800 & 1.0300 & 0.9600 \\
\hline$T_{p 39-57}$ & 0.9900 & 1.0400 & 0.9800 & 1.0400 & 1.0200 & 0.9400 \\
\hline$Y_{h 18}$ & 0.0900 & 0.0500 & 0.0250 & 0.0300 & 0.1000 & 0.0700 \\
\hline$Y_{h 25}$ & 0.1000 & 0.0800 & 0.1000 & 0.0600 & 0.1000 & 0.0650 \\
\hline$Y_{h 53}$ & 0.0800 & 0.1000 & 0.1000 & 0.1000 & 0.0700 & 0.0650 \\
\hline Fitness $f$ & 15426 & 15435 & 15436 & 15443 & 15453 & 15470 \\
\hline$C_{i}(\$)$ & 15407 & 15415 & 15414 & 15420 & 15429 & 15448 \\
\hline$P_{\text {Loss }}(M W)$ & 17.1611 & 17.2709 & 19.1394 & 19.6284 & 18.4116 & 19.6433 \\
\hline$\sum\left|V_{i}-V_{\text {ref }}\right|$ & 2.3280 & 2.6581 & 2.7262 & 2.6219 & 2.8305 & 2.1472 \\
\hline
\end{tabular}

where $f$ is the best fitness of each trial, $f_{\max }$ and $f_{\min }$ are the maximum and minimum fitness, respectively, among 30 trials. $n$ is the number of trials.

As seen in Table 6, in the 26-bus system, as compared with those obtained by other stochastic methods, the performance indexes obtained by the proposed SCPSO method, $\Delta f=35, \mu=15829$, and $\sigma=14.3111$, are obviously better. In the IEEE 57-bus system, as compared with those obtained by other PSO methods, the performance indexes obtained by the proposed SCPSO method, $\Delta f=78, \mu=15447$, and $\sigma=16.1596$, are also obviously better.

In addition, as shown by Table 5, the proposed SCPSO method is still the most outstanding method in terms of fitness, generation cost, transmission loss, and bus-voltage deviation. For example, in the 26-bus system, the SCPSO method has the best fitness of 15812, thus implying a total generation cost of $\$ 15,487$, a transmission loss of $11.6166 \mathrm{MW}$, and a summation of bus-voltage deviation of $0.3432 \mathrm{pu}$. In the IEEE 57 -bus system, the SCPSO method has the best fitness of 15426, thus implying a total generation cost of $\$ 15,407$, a transmission loss of $17.1611 \mathrm{MW}$, and a summation of bus-voltage deviation of $2.3280 \mathrm{pu}$. These results have shown that the proposed SCPSO method can obtain better solution quality.

5.5. Discussion. Figures 6(a)-6(b) present the convergence tendency using different stochastic methods for showing further the advantages of the proposed SCPSO method. The convergence tendency of average fitness of each proposed method can be found in the 30 trials. As seen in both figures, the proposed SCPSO method has the best convergence behavior that can escape the local optima. Specially, the SCPSO method is superior to the CPSO method because the former has the simplex operator that can avoid being trapped in local minima.

System operations must know which line or generation outages will cause power flows or voltages to fall outside limits. To verify the feasibility of the solution obtained by the SCPSO method, two profiles of bus voltage are employed and shown in Figure 7. One is a study system under normal operation, denoted by the circle symbol, and the other is a study system under post-contingency conditions, denoted by the cross symbol. Contingency analysis procedures single out failure events such as one-line outage in a power system. The proposed SCPSO-based OPF method is used to check the security constraints. For each outage tested, it checks all lines and voltages against their respective limits. For two study cases, tested systems can work under security constraints including the generation limit, transmission capacity limit, transformer-tap setting limit, and capacitor capacity limit, as shown in Tables 6(a) and 6(b). In the 26-bus system, as can be seen, the optimal settings of control variables obtained by the proposed SCPSO method can still maintain the least possible deviation of bus voltage even when line $\mathrm{L}_{2-7}$ faulted. In the IEEE 57-bus system, the same phenomenon was obtained by the proposed SCPSO method when line $\mathrm{L}_{1-17}$ faulted. The results show that the optimal settings of control variables allow systems to be operated defensively. 


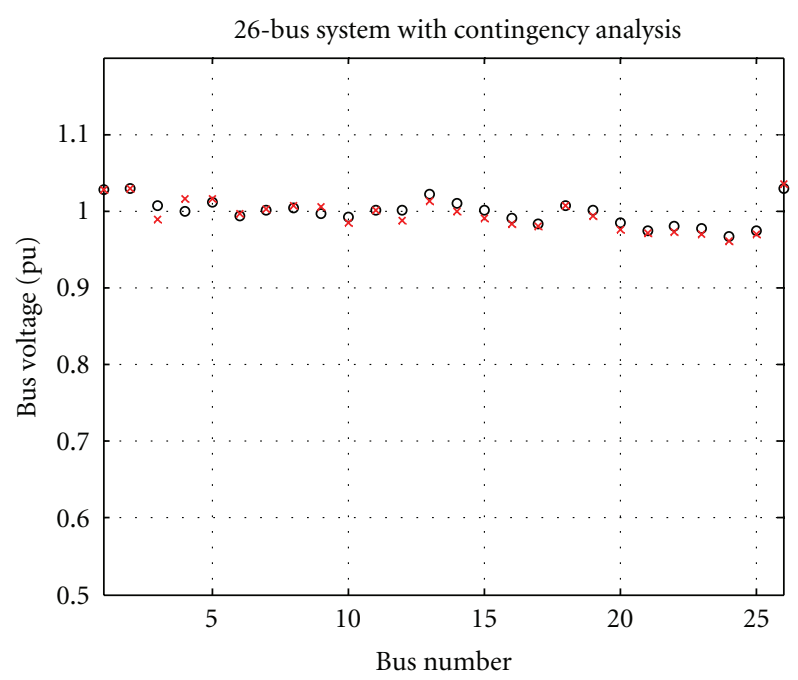

O Normal operation

$\times$ Post-contingency

(a)

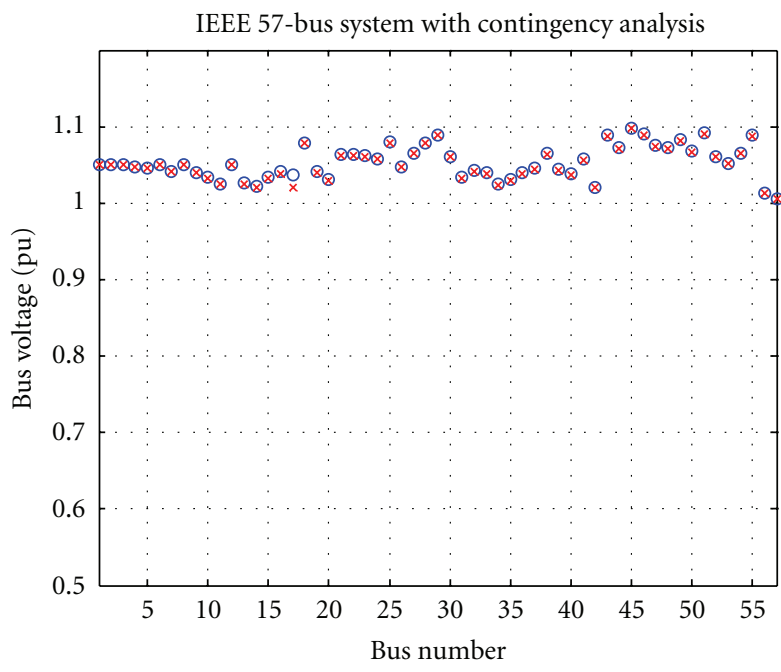

O Normal operation

$\times$ Post-contingency

FIGURE 7: Bus voltage profiles of study systems. (a) Bus voltage profile of system in 26-bus system. (b) Bus voltage profile of system in IEEE 57-bus system.

\section{Conclusion}

In this paper, an associated objective of CC-OPF is defined to be capable of minimizing the total generation cost as well as enhancing the security of the system even if the system suffers transmission line outages. For effectively solving the CC-OPF problem, a chaotic particle swarm optimization with simplex operator (SCPSO) is presented. The proposed SCPSO method, which involves the chaotic map and the downhill simplex search, can avoid the premature convergence of PSO and escape local minima. As shown in various comparisons, the solutions obtained by the proposed SCPSO method are superior to those obtained by other stochastic techniques in terms of solution quality and convergence characteristic.

Our main work in the future is to find out a more efficient parameter control method to verify further the advantages of the proposed SCPSO method in solving large-scale CC-OPF and security-constrained OPF problems.

\section{Acknowledgments}

The authors gratefully acknowledge the financial support from the National Science Council of Republic of China under contract NSC94-2218-E-244-003 and the technical support from Taiwan Power Company.

\section{References}

[1] A. J. Wood and B. F. Wollenberg, Power Generation Operation and Control, John Wiley \& Sons, New York, NY, USA, 1996.

[2] L. L. Lai, J. T. Ma, R. Yokoyama, and M. Zhao, "Improved genetic algorithms for optimal power flow under both normal and contingent operation States," International Journal of
Electrical Power and Energy Systems, vol. 19, no. 5, pp. 287292, 1997.

[3] B. Stott, O. Alsac, and A. J. Monticelli, "Security analysis and optimization," Proceedings of the IEEE, vol. 75, no. 12, pp. 1623-1644, 1987.

[4] H. Harsan, N. Hadjsaid, and P. Pruvot, "Cyclic security analysis for security constrained optimal power flow," IEEE Transactions on Power Systems, vol. 12, no. 2, pp. 948-953, 1997.

[5] H. Yoshida, K. Kawata, Y. Fukuyama, S. Takayama, and Y. Nakanishi, "A Particle swarm optimization for reactive power and voltage control considering voltage security assessment," IEEE Transactions on Power Systems, vol. 15, no. 4, pp. 12321239, 2000.

[6] E. Liu, A. D. Papalexopoulos, and W. F. Tinney, "Discrete shunt controls in a Newton optimal power flow," IEEE Transactions on Power Systems, vol. 7, pp. 1519-1528, 1999.

[7] D. C. Walters and G. B. Sheble, "Genetic algorithm solution of economic dispatch with value point loading," IEEE Transactions on Power Systems, vol. 8, no. 3, pp. 1325-1332, 1993.

[8] A. G. Bakirtzis, P. N. Biskas, C. E. Zoumas, and V. Petridis, "Optimal power flow by enhanced genetic algorithm," IEEE Transactions on Power Systems, vol. 17, no. 2, pp. 229-236, 2002.

[9] D. Devaraj and B. Yegnanarayana, "Genetic-algorithm-based optimal power flow for security enhancement," IEE Proceedings- Generation, Transmission and Distribution, vol. 152, no. 6, pp. 899-905, 2005.

[10] M. Todorovski and D. Rajičić, "An initialization procedure in solving optimal power flow by genetic algorithm," IEEE Transactions on Power Systems, vol. 21, no. 2, pp. 480-487, 2006.

[11] M. Younes, M. Rahli, and L. Abdelhakem-Koridak, "Optimal Power Flow based on Hybrid Genetic Algorithm," Journal of 
Information Science and Engineering, vol. 23, no. 6, pp. 18011816, 2007.

[12] M. A. Abido, "Optimal power flow using tabu search algorithm," Electric Power Components and Systems, vol. 30, no. 5, pp. 469-483, 2002.

[13] T. Kulworawanichpong and S. Sujitjorn, "Optimal power flow using tabu search," IEEE Power Engineering Review, vol. 22, no. 6, pp. 37-40, 2002.

[14] H. R. Cai, C. Y. Chung, and K. P. Wong, "Application of differential evolution algorithm for transient stability constrained optimal power flow," IEEE Transactions on Power Systems, vol. 23, no. 2, pp. 719-728, 2008.

[15] C. Y. Chung, C. H. Liang, K. P. Wong, and X. Z. Duan, "Hybrid algorithm of differential evolution and evolutionary programming for optimal reactive power flow," IET Generation, Transmission and Distribution, vol. 4, no. 1, pp. 84-93, 2010.

[16] A. A. Abou El Ela, M. A. Abido, and S. R. Spea, "Optimal power flow using differential evolution algorithm," Electric Power Systems Research, vol. 80, no. 7, pp. 878-885, 2010.

[17] M. A. Abido, "Optimal power flow using particle swarm optimization," International Journal of Electrical Power and Energy Systems, vol. 24, no. 7, pp. 563-571, 2002.

[18] A. A. A. Esmin, G. Lambert-Torres, and A. C. Zambroni de Souza, "A hybrid particle swarm optimization applied to loss power minimization," IEEE Transactions on Power Systems, vol. 20, no. 2, pp. 859-866, 2005.

[19] N. Mo, Z. Y. Zou, K. W. Chan, and T. Y. G. Pong, "Transient stability constrained optimal power flow using particle swarm optimisation," IET Generation, Transmission and Distribution, vol. 1, no. 3, pp. 476-483, 2007.

[20] M. R. AlRashidi and M. E. El-Hawary, "Hybrid particle swarm optimization approach for solving the discrete OPF problem considering the valve loading effects," IEEE Transactions on Power Systems, vol. 22, no. 4, pp. 2030-2038, 2007.

[21] P. E. O. Yumbla, J. M. Ramirez, and C. A. Coello Coello, "Optimal power flow subject to security constraints solved with a particle swarm optimizer," IEEE Transactions on Power Systems, vol. 23, no. 1, pp. 33-40, 2008.

[22] J. B. Park, Y. W. Jeong, J. R. Shin, and K. Y. Lee, "An improved particle swarm optimization for nonconvex economic dispatch problems," IEEE Transactions on Power Systems, vol. 25, no. 1, pp. 156-166, 2010.

[23] J. Kennedy and R. Eberhart, "Particle swarm optimization," in Proceedings of IEEE International Conference on Neural Netwroks, vol. 4, pp. 1942-1948, Perth, Australia, November 1995.

[24] Y. Shi and R. C. Eberhart, "Empirical study of particle swarm optimization," in Proceedings of the Congress on Evolutionary Computation, vol. 3, pp. 1945-1950, Piscataway, NJ, USA, July 1999.

[25] A. Stacey, M. Jancic, and I. Grundy, "Particle swarm optimization with mutation," in Proceedings of IEEE International Conference on Evolutionary Computation, vol. 2, pp. 14251430, December 2003.

[26] N. Higashi and H. Iba, "Particle swarm optimization with Gaussian mutation," in Proceedings of the IEEE on Swarm Intelligence Symposium, pp. 72-79, April 2003.

[27] B. Liu, L. Wang, Y. H. Jin, F. Tang, and D. X. Huang, "Improved particle swarm optimization combined with chaos," Chaos, Solitons and Fractals, vol. 25, no. 5, pp. 1261-1271, 2005.

[28] L. D. S. Coelho, "A quantum particle swarm optimizer with chaotic mutation operator," Chaos, Solitons and Fractals, vol. 37, no. 5, pp. 1409-1418, 2008.
[29] H. Liu, "An adaptive chaotic particle swarm optimization," in Proceedings of the 2nd ISECS International Colloquium on Computing, Communication, Control, and Management (CCCM '09), pp. 254-257, August 2009.

[30] K. Tatsumi, H. Yamamoto, and T. Tanino, "A perturbation based chaotic particle swarm optimization using multitype swarms," in Proceedings of International Conference on Instrumentation, Control and Information Technology, SICE Annual Conference, pp. 1199-1203, Osaka, Japan, August 2008.

[31] Y. He, J. Zhou, C. Li, J. Yang, and Q. Li, "A precise chaotic particle swarm optimization algorithm based on improved tent map," in Proceedings of the 4th International Conference on Natural Computation (ICNC'08), pp. 569-573, October 2008.

[32] J. A. Nelder and R. Mead, "A simplex method for function minimization," Computer Journal, vol. 7, pp. 308-313, 1965.

[33] V. Torczon, "On the convergence of pattern search algorithms," SIAM Journal on Optimization, vol. 7, no. 1, pp. 123145, 1997.

[34] A. R. Hedar and M. Fukushima, "Hybrid simulated annealing and direct search method for nonlinear unconstrained global optimization," Optimization Methods and Software, vol. 17, no. 5, pp. 891-912, 2002.

[35] S. K. S. Fan, Y. C. Liang, and E. Zahara, "Hybrid simplex search and particle swarm optimization for the global optimization of multimodal functions," Engineering Optimization, vol. 36, no. 4, pp. 401-418, 2004. 

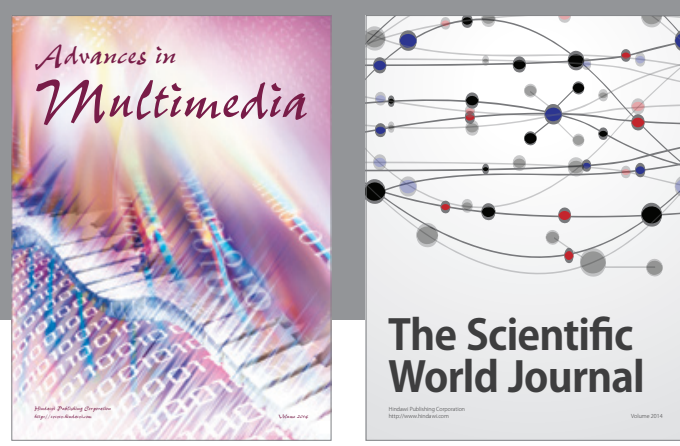

The Scientific World Journal
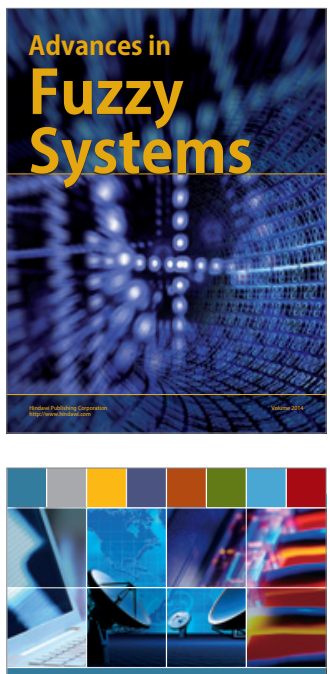

Computer Networks and Communications
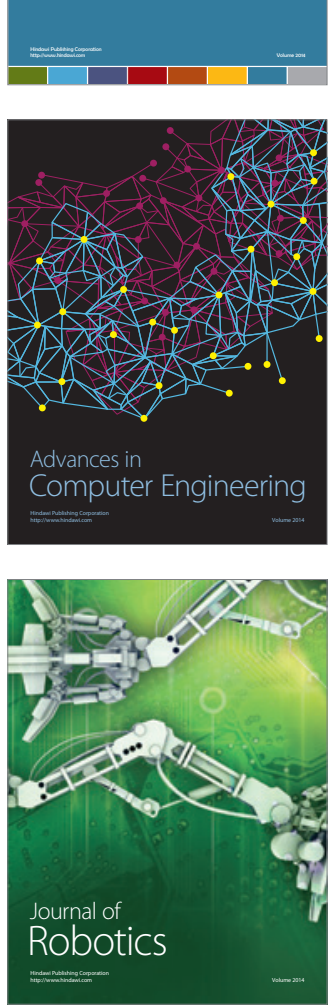
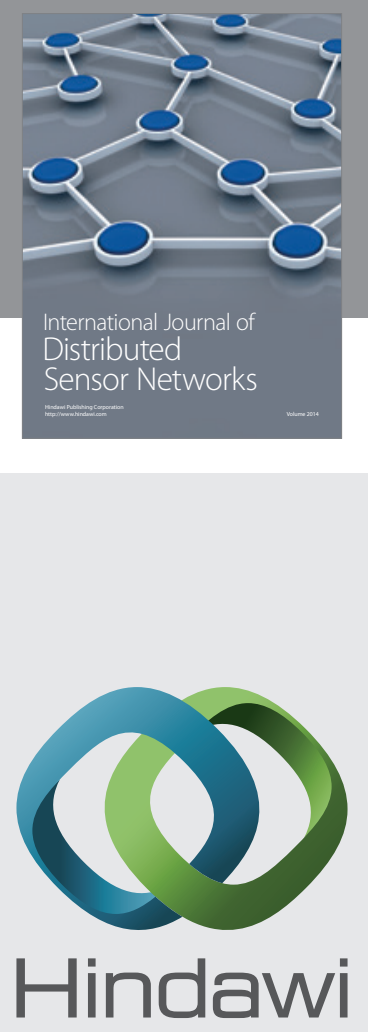

Submit your manuscripts at

http://www.hindawi.com
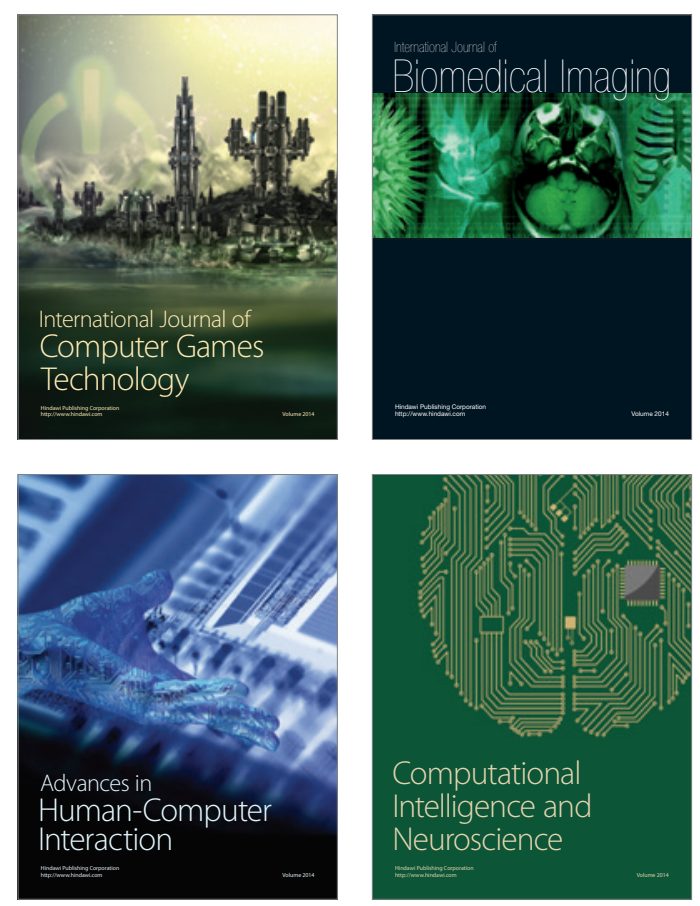
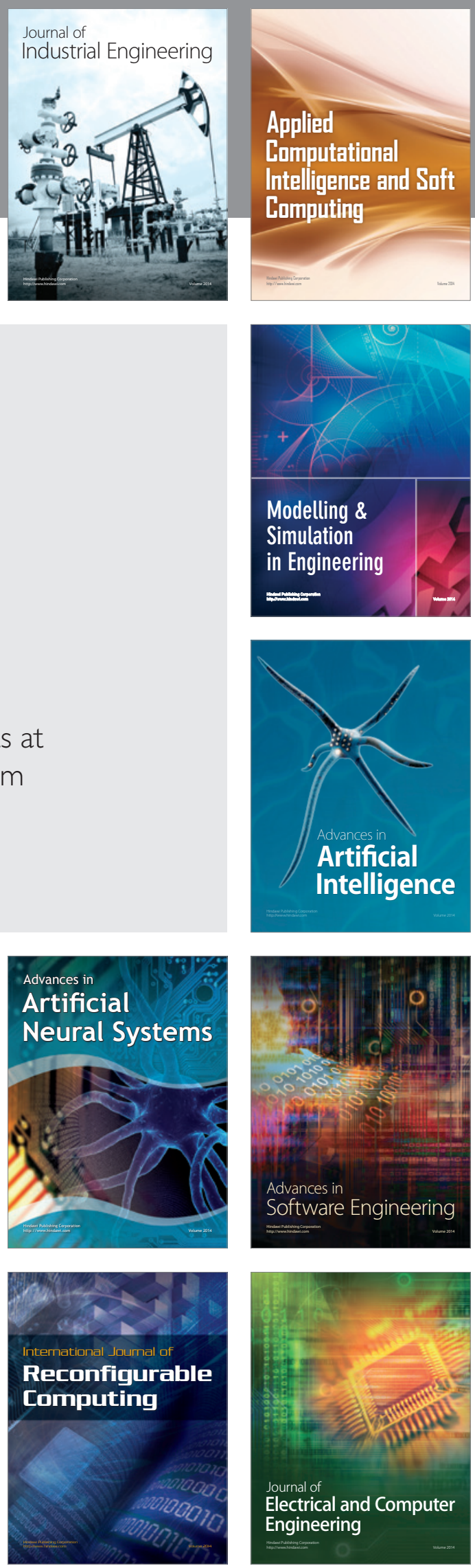